\title{
Quantitative Assessment of Osteoarthritic Knee Instability: Comparison with Conventional Imaging Modalities
}

\author{
V.K.O. Virtanen ${ }^{1 \mathrm{a}}$, J. Thevenot ${ }^{1}$, A. Tiulpin ${ }^{1}$, J. Hirvasniemi ${ }^{1}$, J. Niinimäki ${ }^{2}$, M. \\ Nevalainen $^{1,2}$ and S. Saarakkala ${ }^{1,2}$ \\ ${ }^{1}$ Research Unit of Medical Imaging, Physics and Technology, University of Oulu, \\ Aapistie 5A, 90014 Oulu, Finland, \\ ${ }^{\mathrm{a}} \mathrm{vesa.k.virtanendoulu.fi}$ \\ ${ }^{2}$ Department of Diagnostic Radiology, Oulu University Hospital, Oulu, Finland
}

\begin{abstract}
Knee osteoarthritis (OA) is the most common musculoskeletal disorder affecting all populations. One common knee OA symptom is instability; thus its assessment could allow diagnosing and following-up of the disease without using conventional imaging techniques, such as plain radiography or magnetic resonance imaging (MRI). Knee kinematic measurements using accelerometers could provide a low-cost and non-invasive option to quantify knee instability. The aim of this study was to assess the relationships between kinematic data, instability parameters derived from the imaging techniques, goniometer-based measurements, and radiological OA stage.

The right knees of 66 females (44-67 years) were examined using MRI, plain radiography, and goniometer-based angle measurement. Kellgren-Lawrence (KL) grade and the joint line convergence angle (JLCA) were determined from the radiographs. Cartilage thickness and OA score (MOAKS) were derived from the MRI. A ratio between lateral and medial cartilage thicknesses was calculated from the average thickness of segmented cartilage over the weight bearing area (MRIratio). Accelerometers attached to thigh and shank were used to record kinematic signals during a one-leg-stand test. Power of the accelerometer signals along the anatomical longitudinal axis $\left(\mathrm{P}_{\text {acc }}\right)$ was used as a measure of knee instability. Finally, Spearman's correlations between the acquired parameters and KL grade / MOAKS scores were calculated. Leave-one-out cross-validation and logistic regression were used to discriminate OA subjects $(\mathrm{KL} \geq 2)$.

All the instability parameters ( $\mathrm{P}_{\mathrm{acc}}, \mathrm{JLCA}$ and $\left.\mathrm{MRI}_{\mathrm{ratio}}\right)$, except the goniometer angle, showed significant correlations with KL grading ( $\mathrm{rho}=0.32-0.64, p<0.01$ ) and MOAKS composite score $(\mathrm{rho}=0.35-0.56, p<0.01)$. Both $\mathrm{P}_{\mathrm{acc}}$ and JLCA showed higher areas under the ROC curve to discriminate $\mathrm{OA}$ ( $\mathrm{AUC}=0.76$ and $\mathrm{AUC}=0.78$ ) than MRIratio and goniometer angle ( $\mathrm{AUC}=0.55$ and $\mathrm{AUC}=0.56$ ). Our results demonstrate the clinical potential of kinematic knee instability measurements using low-cost accelerometers. Such approach could become a potential new tool in OA diagnostics.
\end{abstract}

Keywords: Osteoarthritis, Knee, Instability, MRI, Radiography, Accelerometer, Kinematics 


\section{Introduction}

Osteoarthritis (OA) is a degenerative joint disease affecting various tissues, such as bone, cartilage and ligaments. It can occur in any joint, but most typically it affects knee, hand and hip joints. OA is one of the leading causes of disability and pain [1], and its impact on healthcare costs are significant [2,3]. Currently used tools for diagnosing OA are conventional radiography and magnetic resonance imaging (MRI) combined with clinical assessment. It is well known that radiography is not sensitive to early OA changes [4]. MRI provides full 3D imaging of all soft tissues of the joint and can detect OA changes earlier than radiography, but its availability is limited in routine healthcare. Consequently, there is a need for novel low cost, non-invasive and sensitive diagnostic tools to assess joint health.

OA progression is associated with biomechanical changes in the affected joints [5, 6]. One of the most clinically relevant change occurring within the knee joint is the increase of instability, which is caused by the wear and tear of the soft tissues, such as cartilage, menisci and ligaments [7]. Eventually, this will cause greater deviation from normal varus-valgus angle of the knee joint [8].

Gait analysis has been used in multiple studies to assess lower limb movement in subjects with knee OA, however the reported findings have been conflicting. For example, the review article by Ornetti et al. [9] concluded that kinematic parameters were mostly insufficient to be considered as valuable outcome measures. Yet, the recent study by van der Straaten et al. [10] found that differences in knee flexion range of motion, stride and gait cycle duration were distinct in subjects with knee OA when compared to healthy subjects.

Development of accurate and sensitive novel tools for clinical purpose is crucial. For instance, kinematic systems with low accuracy might fail to detect symptomatic changes during movements. Recent progress in electronics enables high accuracy inertial measurement unit (IMU) sensors to be accessible even to the general public. In principle, the kinematic data obtained from these sensor units can provide new insights of the alterations within the knee joints of OA subjects when compared to subjects with healthy knees.

The aim of this study was to assess the feasibility of using low-cost accelerometerbased measurements from thigh and shank to extract information on knee instability, and compare it with currently used clinical modalities, i.e., conventional radiography and MRI. Our study has the following novelties: 1) utilizing the power of accelerometer signal as a parameter for assessing knee instability, and 2) high number of subjects and data from clinical assessment, radiographs and MRI scans to provide a reliable comparison. 


\section{Materials and Methods}

\subsection{Data acquisition protocol and OA staging}

Sixty-six females (57.8 \pm 6.2 years old, range $44-67$ years) with body mass index (BMI) of $27.4 \pm 4.8 \mathrm{~kg} / \mathrm{m} 2$ underwent a clinical trial at Oulu University Hospital, Finland (clinicaltrials.gov study identifier: NCT02937064). The first part of the trial included a background questionnaire, physical examination and varus-valgus angle assessment using a goniometer. For the goniometer measurements, the subjects stood up with legs rotated 10 degrees out. The second part of the trial protocol consisted of knee MRI, anthropometric measurements (weight, height), inertial measurements using kinematic modules (accelerometer and gyroscope), and a weight bearing knee radiograph acquisition. The OA stage was assessed from the radiographs using the KellgrenLawrence (KL) grading scale [11], and from the MRI data using the MRI OA knee scoring (MOAKS) system [12]. A composite MOAKS score was calculated by combining full-thickness and non-full-thickness cartilage degeneration parameters from medial and lateral sides of both femur and tibia, which resulted in a total score between 0 and 24 .

\subsection{Accelerometer analysis}

The accelerometer measurements were performed using a custom-made prototype device with the sensors attached to the thigh and shank as indicated by red marks in Figure 1A. One-leg-stand movement was repeated twice. Acceleration in anatomical longitudinal axis was used as a measure of knee instability and the power of the signal $\left(\mathrm{P}_{\mathrm{acc}}\right)$ over the two repeated movements was calculated.

\subsection{Radiograph analysis}

Knee radiographs were acquired using Digital Diagnost (Philips Medical Systems) Xray device with $60 \mathrm{kVp}$ voltage, automatic exposure and pixel size of $0.148 \mathrm{~mm}$ x 0.148 $\mathrm{mm}$. The subjects were instructed to stand on pre-determined feet markers and were leaning their legs against a plate to standardize the degree of knee bending. Joint line convergence angle (JLCA) [13] was calculated from the radiographs (Figure 1B). The required anatomical reference points were manually marked and the angle was calculated with an ad-hoc MATLAB-based (MATLAB R2017B, version 9.2.0.518641, The MathWorks, Inc., Natick, Massachusetts, United States) script. The markings were repeated twice, and the average of both calculated angles was used as the final JLCA value.

\subsection{MRI analysis of cartilage thickness}

MRI images were acquired using Siemens Skyra 3-Tesla scanner using 3D Double Echo Steady State sequence with water excitation (3D-DESS-WE): repetition time of $14.1 \mathrm{~ms}$, echo time of $5 \mathrm{~ms}$, and echo train length of 2 . Slices were oriented in sagittal 
plane, and both the pixel spacing and slice thickness were $0.6 \mathrm{~mm}$. The cartilage was then semi-automatically segmented from the slices using the Mimics software (version 17.0.0.435, Materialise NV, Leuven, Belgium). Lateral and medial compartments of tibial and femoral cartilage was segmented using the following method: the start and end slices were determined by the first slices in which the ligaments were not interfering with cartilage segmentation, except on last slices of medial side where the last slice was determined by visually assessing that enough of the tibial cartilage was still visible in the slice.

To reach high accuracy of the segmentation, a preliminary mask was carefully drawn following the cartilage surface, to solely include cartilage and bone. Subsequently, a thresholding value was manually selected to provide the best compromise to separate cartilage from bone. All the segmented slices were visually checked by the user and corrected if necessary. The end result consisted of four distinct masks corresponding to medial and lateral compartments of the femur and tibia (Figure 1C). The average thickness over the weight-bearing area from the segmented masks for each subject was calculated with a custom-made MATLAB script. The area used for calculations is illustrated in Figure 1C.

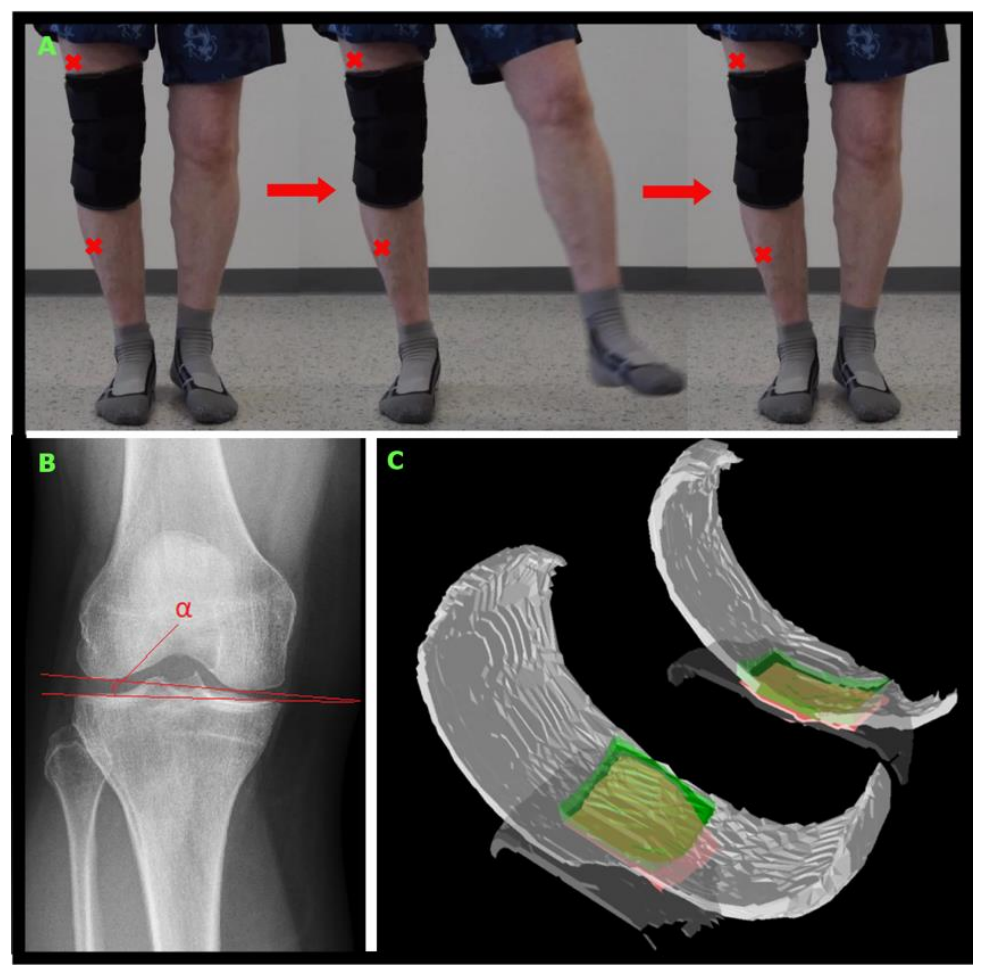

Fig. 1. Visualization of the knee instability parameters' acquisition methods. A) Kinematic power of signal was recorded during one leg stand. Red crosses are marking roughly the placement of sensors; B) Method for calculating JLCA angle ( $\alpha$ ) from plain radiograph; C) 3D cartilage masks with colored areas (green: femur, red: tibia) used in weight bearing area thickness calculations. 


\subsection{Statistical methods}

As a measure of instability, we normalized the data by assessing the relative deviation between medial and lateral sides for all modalities. Briefly, MRI ratio was calculated by dividing higher (between medial and lateral) cartilage thickness by the lower one. Similarly, the absolute value of JLCA was used as a measure of deviation. For the goniometer data, the absolute values of the measured varus or valgus angles were used.

Spearman's correlations were calculated between kinematic instability parameters and OA stages (KL grades and MOAKS-derived composite scores). We also used leave-one-out cross-validation (LOOCV) with logistic regression and performed receiver operating characteristic (ROC) analysis by calculating area under the curve (AUC) to discriminate $\mathrm{OA}$ cases $(\mathrm{KL} \geq 2)$ from controls.

\section{$3 \quad$ Results}

The KL assessment of the knees yielded the following results: KL0: 7, KL1: 25, KL2: 16, KL3: 16, KL4: 2, which corresponds to 32 controls and 34 OA cases. The relation between KL grade and composite MOAKS scores can be seen from the Figure 2.

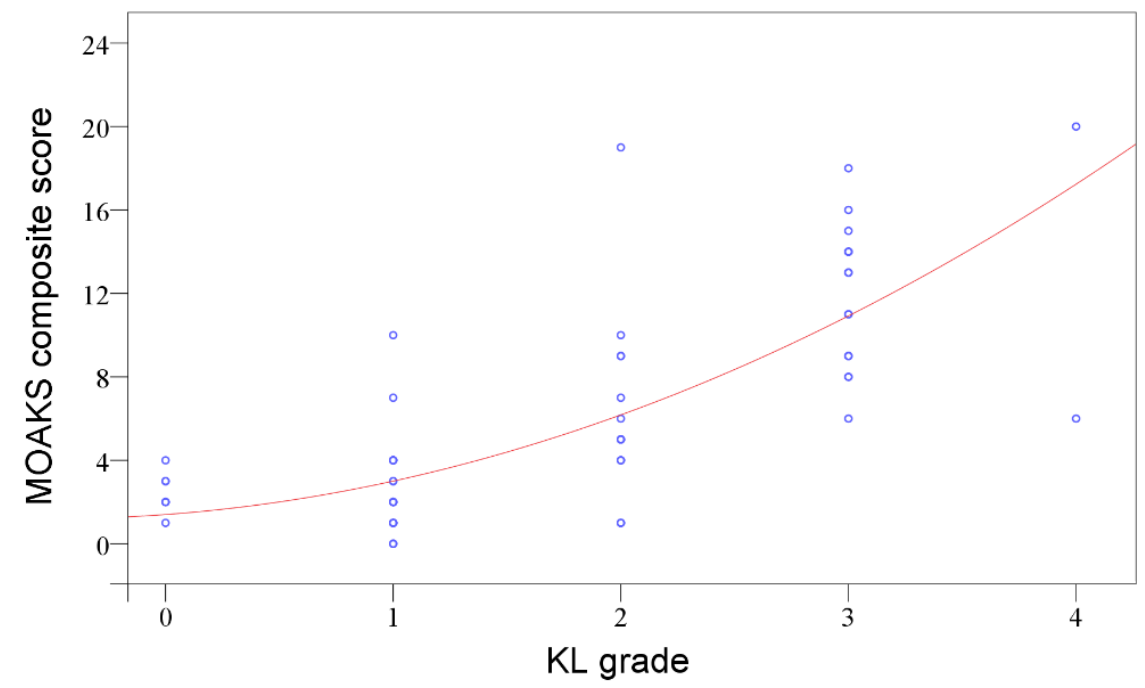

Fig. 2. MOAKS composite score compared to KL grade. The subjects are shown in blue and the second order polynomial fit in red $\left(\mathrm{r}^{2}=0.57\right)$.

Statistically significant correlations $(p<0.01)$ were found for $\mathrm{P}_{\text {acc }}$, JLCA and MRI ratio $_{\text {r }}$ when compared against KL grades or MOAKS composite scores, as shown in Table 1. Compared to KL grade, JLCA achieved a rho of 0.64 , while $\mathrm{P}_{\mathrm{acc}}$ had rho of 0.48 and MRI $_{\text {ratio }}$ had rho of 0.32 . When assessing correlations with MOAKS composite score, 
$\mathrm{P}_{\text {acc }}$ and JLCA achieved similar correlations (rho of 0.56) while MRI $\mathrm{I}_{\text {ratio }}$ had the lowest (rho of 0.35).

Logistic regression with LOOCV yielded AUC of 0.76 (95\% CI: 0.64-0.88) for $\mathrm{P}_{\mathrm{acc}}$

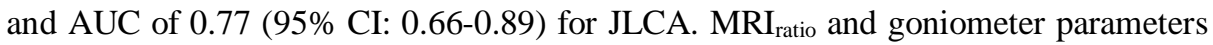
had poor AUC close to 0.5 .

Table 1. Comparison between measured kinematic parameters and radiological findings (Kellgren-Lawrence (KL) grades and composite MOAKS scores), using Spearman correlation and area under the ROC -curve (AUC) for leave-one-out cross validation (LOOCV). $\mathrm{P}_{\text {acc: }}$ power of acceleration signal; JLCA: joint line convergence angle; MRI $\mathrm{I}_{\text {ratio: }}$ ratio between medial and lateral side knee cartilage thickness.

\begin{tabular}{llll}
\hline Parameter & Spearman rho (KL) & Spearman rho (MOAKS) & AUC (LOOCV) \\
\hline$P_{\text {acc }}$ & $0.48^{* *}$ & $0.56^{* *}$ & 0.76 \\
JLCA & $0.64^{* *}$ & $0.56^{* *}$ & 0.77 \\
MRI $_{\text {ratio }}$ & $0.32^{* *}$ & $0.35^{* *}$ & 0.55 \\
Goniometer angle & 0.20 & 0.12 & 0.54 \\
\hline
\end{tabular}

$* p<0.05, * * p<0.01$

\section{Discussion}

Knee instability is known to be associated with OA [6]. As the disease progresses, the alteration of the soft tissues within the joint can result in a knee misalignment and an increased shear and motion during bending. The aim of this study was to assess the feasibility of using low-cost accelerometer-based measurements from thigh and shank to extract information on knee instability and compare it with OA severity evaluated from both conventional radiography and MRI.

Changes in varus-valgus angle have been shown to be associated with OA progression $[8,14,15]$. The greater deviation from a normal angle can be considered as a measure of instability. In the current study, the misalignment of the knee was assessed from two different clinical imaging modalities as well as using a traditional goniometer device. Goniometer is widely used in clinical practice and has been applied in OA studies [16], however despite the measurement being fast and easy to perform, its reliability to define the varus-valgus angle is low [17]. Conventional knee radiography allows to determine the actual anatomical angle between the femur and the tibia (an angle close to the one assessed by goniometer), but here we decided to focus on the JLCA as it is more representative of the difference in cartilage thicknesses between medial and lateral sides. We also measured the ratio between medial and lateral cartilage thicknesses segmented volumetrically from MRI scans of unloaded knees, as an analogic evaluation of the joint space width difference between sides, comparable to the JLCA information obtained from the radiographs.

Accelerometers and IMUs have been used in studies related to knee OA, but those have focused on knee range of motion and knee angle during both stance and swing 
phase of gait, as well as temporal parameters of gait during level walking [10]. In our study, we wanted to assess knee instability and hypothesized a one-leg-stand test as a potential experiment to evaluate instability. Due to the age of subjects, lifting of the leg was only done conservatively as shown in the figure 1A, which allowed all of the subjects to perform the test. The test only takes a few seconds to perform and the results can be obtained instantly and objectively, unlike with current clinical modalities which require subjective assessment from the data. The results show that the $\mathrm{P}_{\text {acc }}$ signal during one-leg-stand test correlates significantly with $\mathrm{OA}$ stage and can discriminate OA patients from control subjects with similar AUC as JLCA.

While both JLCA and $\mathrm{MRI}_{\mathrm{ratio}}$ are estimations of the difference between the lateral and medial side, the obtained results showed that JLCA outperformed the MRI ratio in assessing OA. One reason of this finding could be that the joint is loaded during radiography and that the compression of the cartilage might be directly affected not only by its original thickness, but also by its composition changing with OA [18]. Furthermore, JLCA is more representative of the overall current joint condition (the surfaces of the bones are used as references), whereas menisci and ligaments were totally ignored in the $\mathrm{MRI}_{\text {ratio }}$ assessment (only cartilage thicknesses were used). While JLCA and $\mathrm{P}_{\text {acc }}$ provided similar correlations with MOAKS composite score, the higher correlation obtained between JLCA and KL grading is most likely because both of those parameters were evaluated from the same radiographs. Moreover, the standardized standing position required during the knee radiography might have accentuated the medial-lateral difference - especially in subjects with high knee joint laxity - resulting in higher correlations with KL grading.

The angles measured by the goniometer did not show any statistically significant association with OA severity. One explanation for the poor performance of this measurement could be that multiple subjects were overweight and/or had knees with inflammation, affecting the accuracy of the measurements as locating the anatomical references can be challenging in these cases. Goniometer measurements were performed by experienced clinicians using established measurement protocols, but the reliability of the method, unfortunately, could not be assessed since only a single measurement per subject was conducted. The results reported here suggest that goniometer measurements may not be an appropriate modality to be used in clinical OA trials.

As a limitation of this study, the protocol for kinematic acquisition had some variation among the subjects: due to anatomical differences, the location of the sensors was not constant between the subjects. We tried to diminish this variation to a minimum by attaching a rigid 3D printed frames with straps to both thigh and shank. This arrangement reduced the artefacts related to loose tissues and skin movements, which are common when sensors are directly taped onto the skin. From this point of view, one-leg-stand test is a good candidate for assessing knee joint instability with accelerometers as it involves minimal movement. A second limitation of the study is related to the cartilage thickness measurements from MRI: while the method we proposed here should provide an accurate segmentation of the tissue, it cannot overcome the low resolution of the modality. Finally, in this study we had only two patients with KL grading of 4, corresponding to severe OA. While a more even 
distribution could have provided better correlations with the disease, in this clinical trial we recruited subjects with early to mild osteoarthritis, as the diagnosis at those stages is more challenging than at later phases. Eventually, the ability of this kinematic approach to discriminate earlier stages of OA further supports the potential of this method in clinical applications.

As a conclusion, the kinematic approach presented in this study provides a simple, low-cost and non-invasive solution to obtain information on the knee joint instability. The preliminary validation of this method suggests the feasibility of using simple kinematics as a potential diagnostic tool for OA. The combination of this instability information with other parameters known to be associated with the condition should be considered in the future to improve the accuracy of the models to discriminate OA in clinical practice.

\section{Acknowledgements}

The authors would like to thank the patients for their participation in the study. The study was funded by the Finnish Funding Agency for Technology and Innovation (TEKES) and Oulu University Hospital.

\section{Conflicts of Interest}

The authors declare that they have no conflict of interest.

\section{References}

1. Chu CR, Millis MB, Olson SA. Osteoarthritis: From Palliation to Prevention: AOA Critical Issues. J Bone Joint Surg Am 96:e130 (2014).

2. Neogi T. The epidemiology and impact of pain in osteoarthritis. Osteoarthritis Cartilage 21:1145-1153 (2013).

3. Hiligsmann M, Cooper C, Arden N, Boers M, Branco JC, Luisa Brandi M, Bruyere O, Guillemin F, Hochberg MC, Hunter DJ, Kanis JA, Kvien TK, Laslop A, Pelletier JP, Pinto D, Reiter-Niesert S, Rizzoli R, Rovati LC, Severens JL, Silverman S, Tsouderos Y, Tugwell P, Reginster JY. Health economics in the field of osteoarthritis: an expert's consensus paper from the European Society for Clinical and Economic Aspects of Osteoporosis and Osteoarthritis (ESCEO). Semin Arthritis Rheum 43:303-313. (2013)

4. Guermazi A, Roemer FW, Burstein D, Hayashi D. Why radiography should no longer be considered a surrogate outcome measure for longitudinal assessment of cartilage in knee osteoarthritis. Arthritis Res Ther 13:247. (2011)

5. Heijink A, Gomoll AH, Madry H, Drobnic M, Filardo G, Espregueira-Mendes J, Van Dijk CN. Biomechanical considerations in the pathogenesis of osteoarthritis of the knee. Knee Surg Sports Traumatol Arthrosc 20:423-435. (2012)

6. Blalock D, Miller A, Tilley M, Wang J. Joint instability and osteoarthritis. Clin Med Insights Arthritis Musculoskelet Disord 8:15-23. (2015) 
7. Lohmander LS, Englund PM, Dahl LL, Roos EM. The long-term consequence of anterior cruciate ligament and meniscus injuries: osteoarthritis. Am J Sports Med 35:1756-1769. (2007)

8. Freisinger GM, Schmitt LC, Wanamaker AB, Siston RA, Chaudhari AMW. Tibiofemoral Osteoarthritis and Varus-Valgus Laxity. J Knee Surg 30:440-451. (2017)

9. Ornetti P, Maillefert JF, Laroche D, Morisset C, Dougados M, Gossec L. Gait analysis as a quantifiable outcome measure in hip or knee osteoarthritis: a systematic review. Joint Bone Spine 77:421-425. (2010)

10. van der Straaten R, De Baets L, Jonkers I, Timmermans A. Mobile assessment of the lower limb kinematics in healthy persons and in persons with degenerative knee disorders: A systematic review. Gait Posture 59:229-241. (2017)

11. KELLGREN JH, LAWRENCE JS. Radiological assessment of osteo-arthrosis. Ann Rheum Dis 16:494-502. (1957)

12. Hunter DJ, Guermazi A, Lo GH, Grainger AJ, Conaghan PG, Boudreau RM, Roemer FW. Evolution of semi-quantitative whole joint assessment of knee OA: MOAKS (MRI Osteoarthritis Knee Score). Osteoarthritis Cartilage 19:990-1002. (2011)

13. Kleeblad LJ, van der List JP, Pearle AD, Fragomen AT, Rozbruch SR. Predicting the Feasibility of Correcting Mechanical Axis in Large Varus Deformities With Unicompartmental Knee Arthroplasty. J Arthroplasty. (2017)

14. Sharma L, Chmiel JS, Almagor O, Felson D, Guermazi A, Roemer F, Lewis CE, Segal $\mathrm{N}$, Torner J, Cooke TD, Hietpas J, Lynch J, Nevitt M. The role of varus and valgus alignment in the initial development of knee cartilage damage by MRI: the MOST study. Ann Rheum Dis 72:235-240. (2013)

15. Bastick AN, Belo JN, Runhaar J, Bierma-Zeinstra SM. What Are the Prognostic Factors for Radiographic Progression of Knee Osteoarthritis? A Meta-analysis. Clin Orthop Relat Res 473:2969-2989. (2015)

16. Amaratunga HA, Adikari SB, Dassanayake TL, Gamage J, Suraweera HJ. Relationship between the goniometric alignment and articular cartilage damage in knee osteoarthritis. Ceylon Med J 62:167-174. (2017)

17. van Trijffel E, van de Pol RJ, Oostendorp RA, Lucas C. Inter-rater reliability for measurement of passive physiological movements in lower extremity joints is generally low: a systematic review. J Physiother 56:223-235. (2010)

18. Julkunen P, Jurvelin JS, Isaksson H. Contribution of tissue composition and structure to mechanical response of articular cartilage under different loading geometries and strain rates. Biomech Model Mechanobiol 9:237-245. (2010) 